This document is the Accepted Manuscript version of a Published Work that

appeared in final form in RSC Adv., 2016, 6, 31210 DOI: 10.1039/c6ra02497a

\title{
Increasing Cell Viability Using Cd-Free - InP/ZnS@silica@Layered Double Hydroxide - Materials for Biological Labelling
}

\author{
Ivan Castelló Serrano, ${ }^{\mathrm{a}, \mathrm{b}}$ Maria Antonietta De Matteis, ${ }^{\mathrm{b}}$ Georgiana Stoica ${ }^{\mathrm{a}, *}$ Emilio Palomares ${ }^{\mathrm{a}, \mathrm{e}^{*}}$ \\ ${ }^{a}$ Institute of Chemical Research of Catalonia (ICIQ), Avinguda del Països Catalans 16, 43007 \\ Tarragona, Spain.Tel: +34 977920200; E-mail: gstoica@iciq.es. \\ ${ }^{b}$ Telethon Institute of Genetics and Medicine (TIGEM), Campi Flegrei 34, 80078 Pozzuoli, Naples, \\ Italy. \\ ${ }^{c}$ Institució Catalana de Recerca I Estudis Avançats (ICREA). Passeig Lluís Companys 23, E-08010 \\ Barcelona, Spain.
}

\begin{abstract}
In this work we describe the synthesis and characterization of InP/ZnS@silica@LDH nanoparticles and, moreover, their use as biomarkers. The use of dual systems combining silica shells and hydrotalcite nanocoatings favors the cell viability in clear contrast with the system with only silica shells. Furthermore, the use of Cd-free luminophores extends the cells lifetime and illustrates the potential of the InP/ZnS@silica@LDH material as biomarker.
\end{abstract}




\section{Introduction}

The use of nanoparticles for biological labeling, drug delivery, cell apoptosis among other applications is of increasing interest in the field of bio-nanoscience. In all cases, the first step that determines the successful application of the material is the cell uptake process. Although the introduction of the material inside the cell can be carried out in an "active" way, which involves energy to go against some gradient or obstructing, in some cases the cellular uptake takes place through the adsorption of the material in a "passive" way, which depends on the cell membrane permeability, which in turn depends on the membrane composition (proteins and lipids); However, the latter case requires that the material size is in a few nanometers range. ${ }^{1-4}$

Quantum dots have been extensively used as biological markers due to their small nanometer size (2$5 \mathrm{~nm}$ ) and their remarkable luminescence properties that are highly tunable due to the quantum nature of the materials as, for example, CdSe or CdTe. However, if the experiment to carry out requires good cell viability, it is desirable to avoid the use of toxic materials including lead, cadmium and tellurium. ${ }^{5}$ As an alternative, heavy metal-free QDs such as $\mathrm{InP} / \mathrm{ZnS}$ quantum dots, ${ }^{6}$ carbon $\operatorname{dots}^{7,8}$ and graphene quantum dots ${ }^{9}$ have attracted much attention. Besides, embedding the QDs into silica shells results appropriated in view of minimizing the impact of the materials composition leaching in the biological media, in particular for bio-labelling and/or drug delivery ${ }^{10}$ Pioneering studies started using silica to coat QDs, leading to applications in many fields, with particular emphasis on molecules carrier and drug delivery systems. ${ }^{11}$ The delivery of molecules inside mammalian cells with the aim of transferring them across the cell membrane into the cytoplasm is a research area of constantly increasing importance in medicine. Direct delivery is generally inefficient and suffers from problems such as enzymatic degradation, poor bioavailability, poor stability, undesirable accumulative effects of the carrier, and many others. ${ }^{12}$ However, a considerable number of studies have been published throughout the last years, ${ }^{13,14}$ concluding that the uptake and cytotoxicity of silica nanoparticles (NPs) is size and cell-type dependent, involving either clathrin- or caveolin-mediated endocytosis. ${ }^{15}$

In order to benefit at maximum of the opportunities offered by the silica NPs in the biomedical fields, it is evident that these particles need to be highly protected, yet displaying excellent cellular uptake and transport features. In the present study, we tackled this aspect by coating the InP/ZnS@silica core shell NPs with layered double hydroxides (LDHs). In the recent years, it has become apparent that LDHs, also known as hydrotalcites (HTs) are an excellent carrier material due to their outstanding properties with respect to the cell membrane transport. ${ }^{12}$ Although the intercalation of molecules by anionic exchange or delamination-restacking decreases their usually positive surface charge, they remain satisfactorily positively charged to facilitate the cellular uptake. ${ }^{16-19}$ The HT can be used as host matrix for drugs, luminescent dyes but also for nanoparticles (QDs, QD@silica nanospheres, silica NPs, nanorods and lanthanides), enhancing their photoluminescence and stability. ${ }^{11,20-26}$

To the best of our knowledge, hydrotalcite-like materials encapsulating luminescent Cd-free QDs@silica nanospheres were employed herein for the first time for cell imaging. Moreover, we further explored the influence of LDHs on the cellular uptake, and narrow the optimal nanoparticle size, and cell cytotoxicity for the HK-2 cell line. 
The InP/ZnS@silica nanospheres (Figure 1A) have been synthesized using previously reported methods. $^{10,} 27$ Thereafter, the nanospheres (17-65 nm) were coated with LDH nanosheets (Figure 1B) leading to QD@silica@LDH nanoparticles with a final diameter from 22 to $70 \mathrm{~nm}$ (please see the Supplementary Information, Figure S1). A uniform layer of approximately 2.5 - $3 \mathrm{~nm}$ LDH nanosheets was deposited on the surface of the QD@silica nanospheres, as indicated by the electron microscopy images Figure 1. These observations are in good agreement with atomic force microscopy results, ${ }^{28,} 29$ which revealed the existence of $\mathrm{LDH}$ nanosheets of an average height profile 1.2-2 $\mathrm{nm}$. Considering this, we can assume the LDH layer on the surface of QD@silica nanospheres corresponds to only a few (two to three) unilamellar nanosheets.

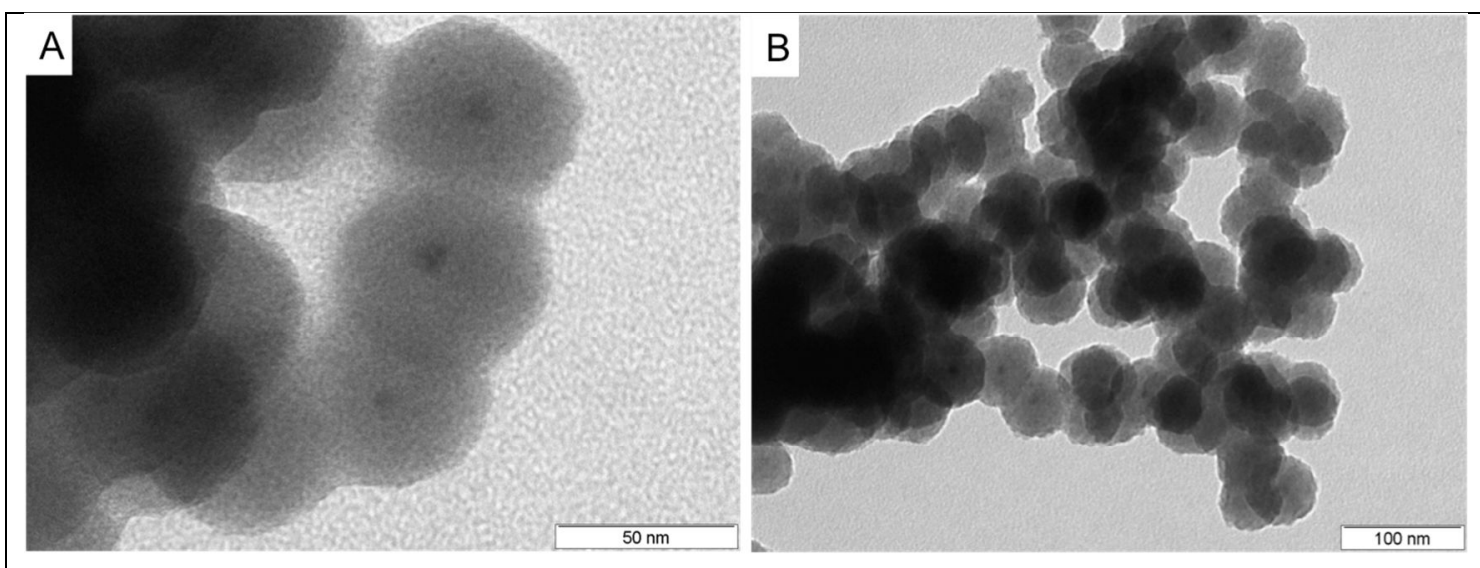

Figure 1. Typical TEM images of $55 \mathrm{~nm}$ original InP/ZnS@silica nanospheres (A) and $60 \mathrm{~nm}$ resulting QD@silica@LDH hybrid nanocomposites (B), respectively.

Figure 2 shows the representative confocal images for the HK-2 cells incubated with the QD@silica@LDH nanoparticles. On the one hand, the nanoparticles with diameters between 32 nm and $40 \mathrm{~nm}$ showed higher cell uptake. On the other hand, larger spheres with diameters between $55 \mathrm{~nm}$ and $65 \mathrm{~nm}$ were introduced in the cells in a lesser extent. Interestingly, smaller nanoparticles $(17 \mathrm{~nm}$ and $27 \mathrm{~nm}$ ) also displayed a lower uptake by the HK-2 cells.

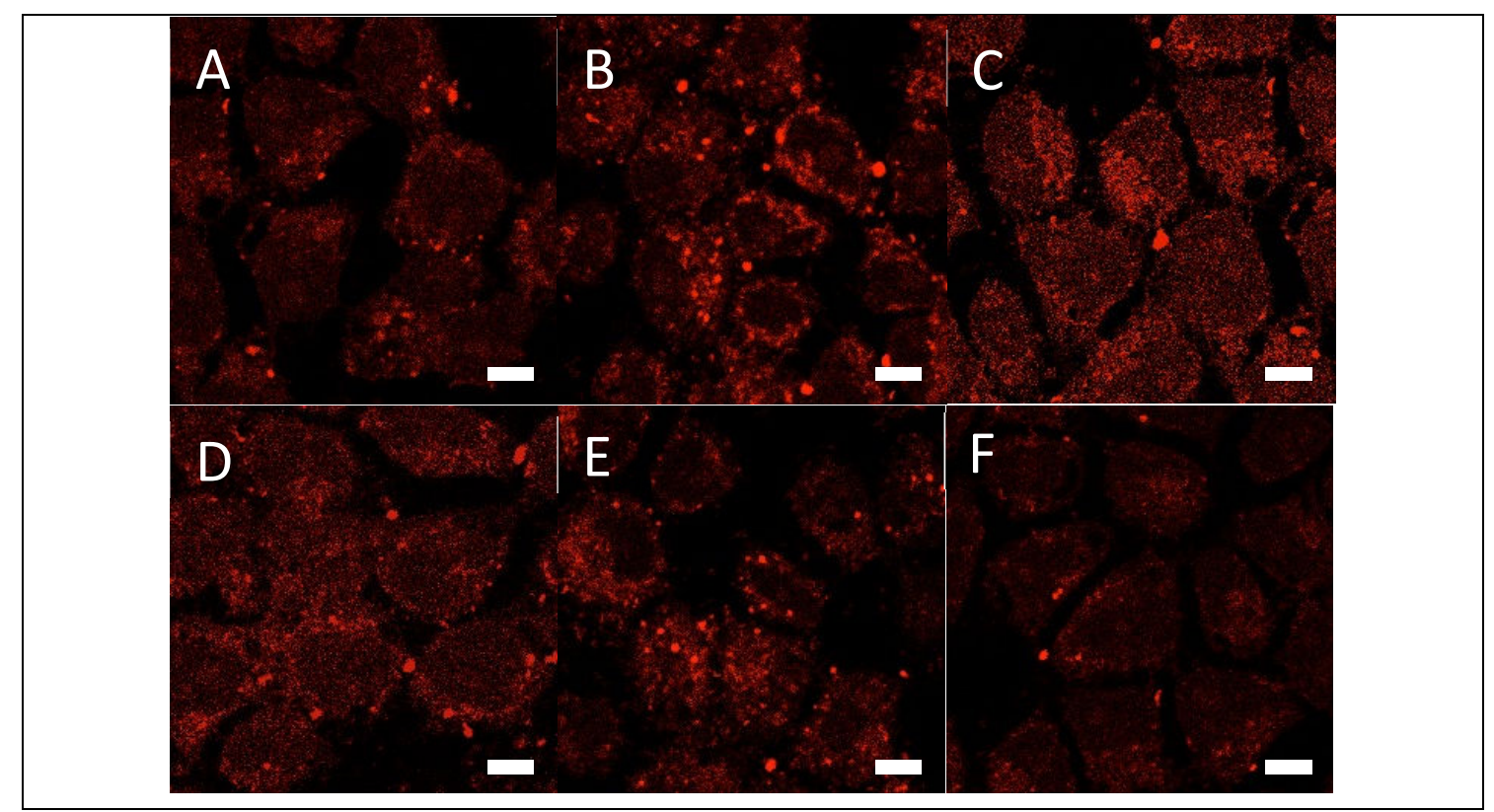


Figure 2. Confocal images of HK-2 cells incubated at $37^{\circ} \mathrm{C}$ with $15 \mathrm{nM}$ of QD@silica@LDH NPs for $30 \mathrm{~min}$ : a) $17 \mathrm{~nm}$, b) $27 \mathrm{~nm}$, c) $32 \mathrm{~nm}$, d) $40 \mathrm{~nm}$, e) $55 \mathrm{~nm}$, and f) $65 \mathrm{~nm}$. The red fluorescence, arising from the $\mathrm{InP} / \mathrm{ZnS}$ inside the NPs, was collected in the range 610-690 nm after excitation at $405 \mathrm{~nm}$ (Fig. S2). The scale bars correspond to $20 \mathrm{~nm}$.

Figure 3 shows the cell culture luminescence intensity versus the nanoparticle size. As it can be seen, the luminescence is higher for nanoparticles with $32 \mathrm{~nm}$ diameter, in good agreement with the higher cell uptake of these particles for the HK-2 cells (Z-stack movies in SI). The HeLa cells were used as control as it is known that these particular type of cells is more robust (less sensitive) towards the use of silica coating nanoparticles. The control and the HK-2 cells show similar behavior which implies that the hydrotalcite coated nanoparticles do not affect different type of cell lines with different sensitivity towards silica coated nanoparticles.

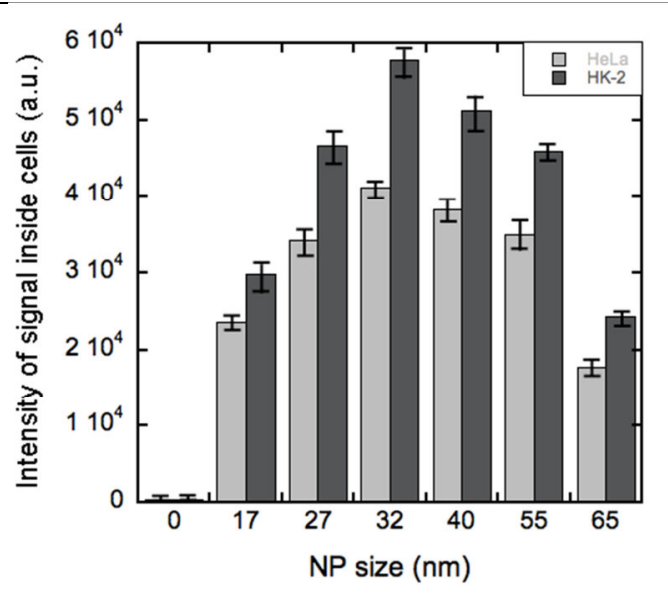

Figure 3. Luminescence emission intensity of QD@silica@LDH nanoparticles in HK-2 (black) and HeLa cells (grey) after 30 min incubation, respectively. The histogram depicts the mean standard error of at least three independent experiments $(n=30)$.

Our results are in line with previously reported experimental studies on targeted drug delivery into cells and uptake of nanoparticles, which have identified the particle size as an important factor in the cellular uptake. Nonetheless, Mou et al. ${ }^{30}$ found that silica nanospheres of $50 \mathrm{~nm}$ in diameter were the most suitable candidate for the cellular uptake of nanoparticles. From our experiments however, we observed that silica nanospheres of $32 \mathrm{~nm}$ are optimal in the case of the HK-2 cell line. Our hypothesis is that the use of the LDH coating improves the cellular uptake. However, in case of nanoparticles smaller than 10 $\mathrm{nm}$ in diameter, the nanoparticles are accumulated on the cell membrane prior to internalization as reported by Treuel et al. ${ }^{31}$ In contrast, our observations using confocal microscopy suggest that for the QD@silica@LDH nanoparticles of 32 nm, after 30 min of incubation, the material is located in the endosomes and cell cytosol (Figure 4), which is a consequence of the LDH shell onto the QD@silica NPs. Xu et al. ${ }^{32}$ confirmed that LDH-based uptake is a type of clathrin-mediated endocytosis, together with a minor contribution of caveole-mediated endocytosis. In the same study, the authors further showed that endocytosed particles are subsequently stored in slowly acidifying vesicles of the endosomal pathway. 
The fact that our material is released from the endosomes to the cell cytoplasm and avoids the lysosomal degradation is a great advantage for bioimaging and delivering systems.

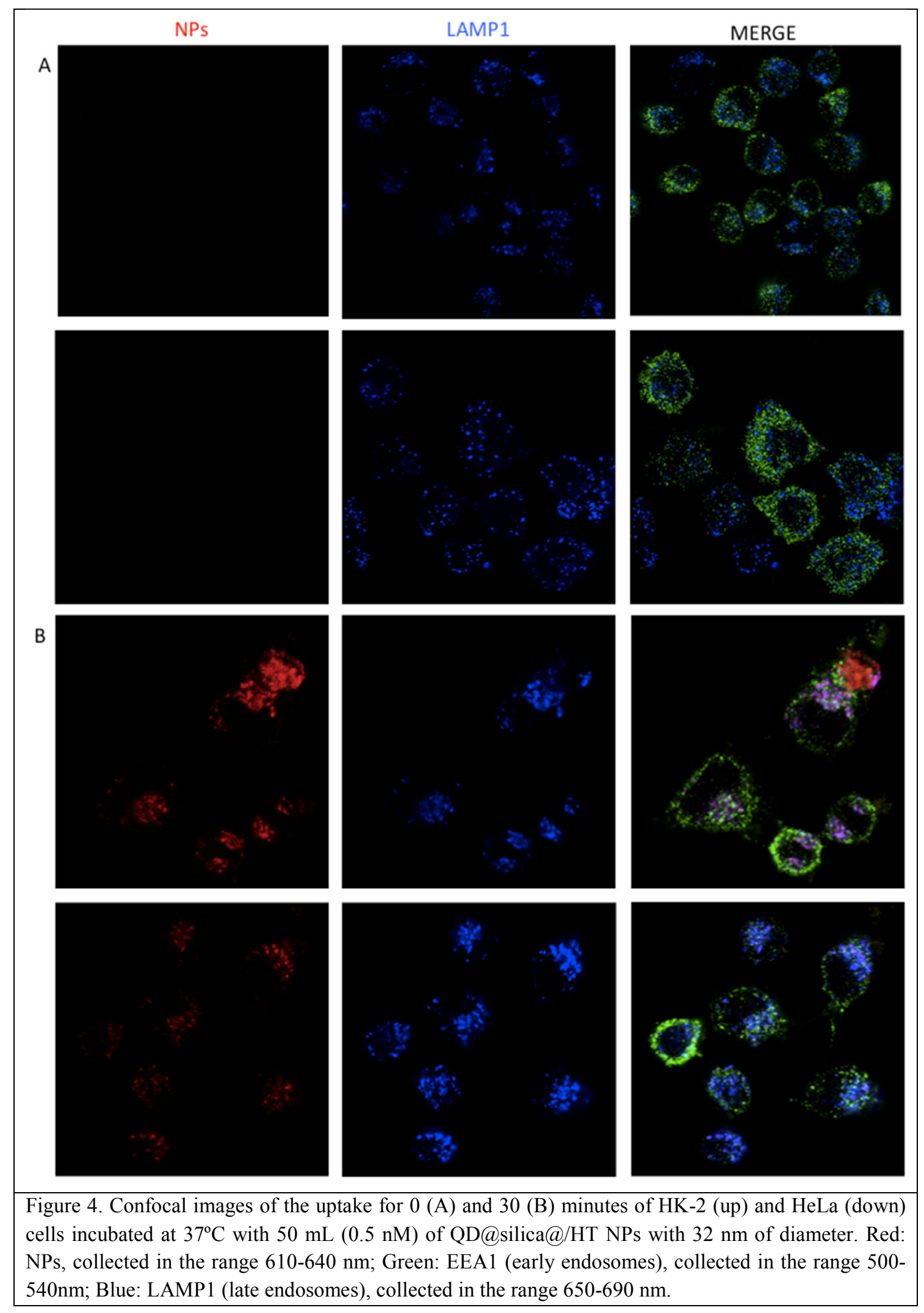


After our experiments of cellular uptake, we further investigated if the use of the hydrotalcite shell on the silica nanospheres has any effect on the cells viability. Previous studies showed that proximal tubular cell lines, among them the HK-2 cell line, were sensitive to the oxidative stress provoked by silica NPs. ${ }^{33}$ The MTT (3-(4,5-dimethylthiazol-2-yl)-2,5-diphenyltetrazolium bromide) tetrazolium reduction assay results (Figure 5) indicate a progressive decrease of the HK-2 cells mitochondrial activity by increasing the number of QD@silica nanoparticles (32 nm, 15nM). Similar remarks have been reported for Human Umbilical Vein Endothelial Cells (HUVEC) cells, also an epithelial cell line, where the silica nanoparticles led to HUVEC cell death, while HeLa cells survived, indicating therefore that HUVEC are highly sensitive to silica exposure. ${ }^{34}$ In clear contrast, the hydrotalcite-coated particles (QD@silica@LDH) exhibited highly satisfactory cell viability in time, leading to the conclusion that the presence of hydrotalcite reduces the cytotoxicity of HK-2 cells.

On the other hand, the uptake kinetics showed again a cell type-dependent behavior (Figure 6), in good agreement with previous studies on silica NPs in different cell types. ${ }^{34,35}$ Moreover, the longer the time of cell exposure to the LDH hybrids, the greater the cellular uptake. Within the first $4 \mathrm{~h}$, the mean number of internalized nanoparticles increased almost linearly for both cell types. Interestingly, after $10 \mathrm{~h}$, the situation was reversed and HeLa cells internalized a larger number of nanoparticles than HK2. Alike behavior was observed for the HUVEC cells upon exposure to silica nanoparticles, thus highlighting that different cellular characteristics, as each cell type has an individual surface property and cellular morphology, display different nanoparticle-cell interactions and uptake processes.

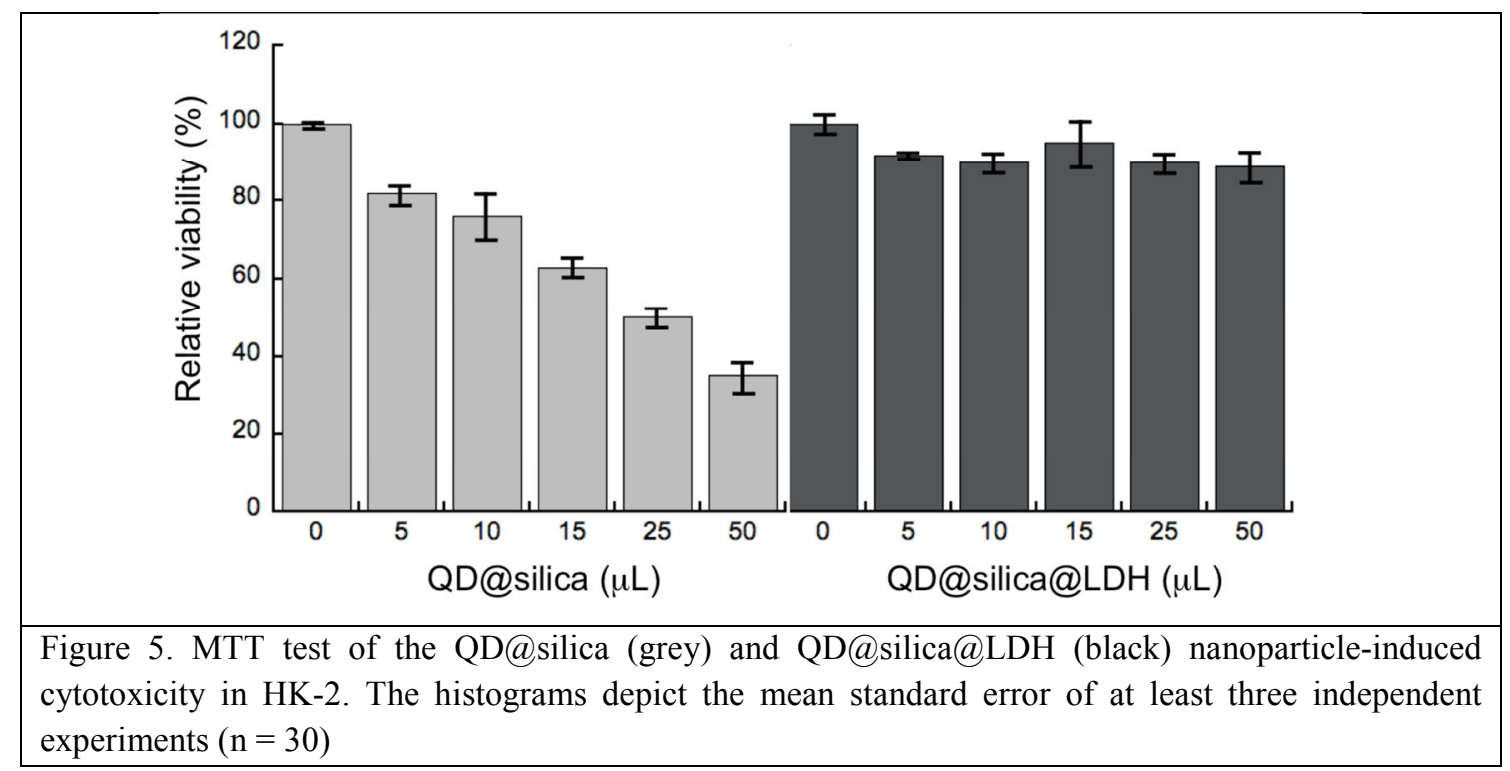




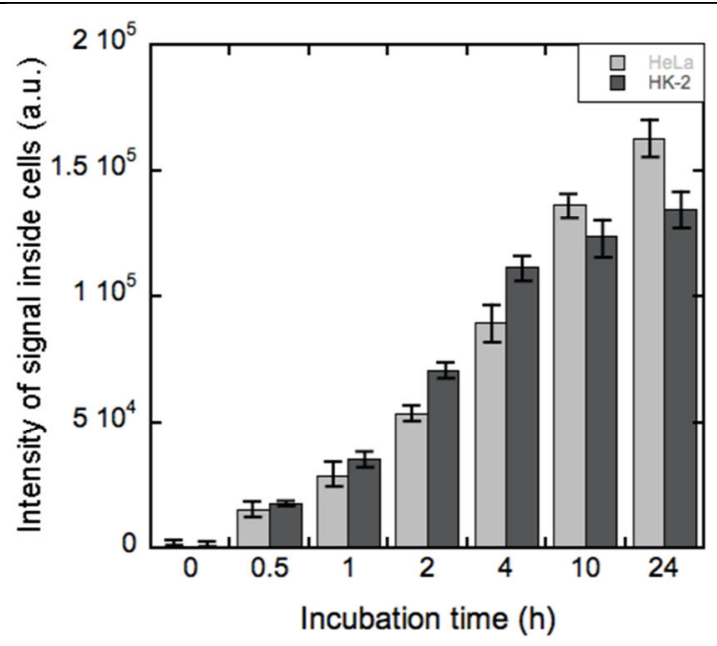

Figure 6. Uptake kinetics of QD@silica@LDH nanoparticles in HeLa (grey) and HK-2 cells (black). The histograms depict the mean standard error of at least three independent experiments $(n=30)$.

As expected from the hydrotalcite uptake mechanism, the endocytosed nanoparticles, stored in the vesicle of the endosomal pathway, are supposed to be released from the endosomes in time, ${ }^{36}$ thus resulting in free QD@silica nanospheres into the cytoplasm. The results in Figure 2 confirmed this behavior. As indicated by the MTT results in Figure 5, the presence of QD@silica should decrease the cell viability of HK-2. Still, the HK-2 cells exposed to QD@silica@LDH exhibit a steady viability 12 h post-uptake, even at higher nanoparticle number. These observations point out to a surprisingly extended lifetime of the QD@silica@LDH composite system, originating hypothetically in the integrity of the LDH layer.

In summary, we demonstrated herein the potential application of highly stable and luminescent $\mathrm{Cd}$-free QD@silica@layered double hydroxide nanospheres for bioimaging. The quick cellular uptake is due to the attractive interactions between the positively charged LDH nanosheets and the negatively charged cell membrane, without further functionalization. Nanoparticles of $32 \mathrm{~nm}$ where uniformly distributed in the cells, indicating this is the optimal size when surrounded by LDH. The coating layer confers a highly biocompatible attribute to the silica NPs, otherwise proved to be toxic by themselves when released into the HK-2, additionally acting like a barrier against degradation. Remarkably, the hydrotalcite shell is a clever strategy to enhance the endosomal escape and thereby increase the stability and lifetime of QD@silica, outstanding features for any bioimaging system.

\section{Acknowledgements}

We would like to thank ICIQ, TIGEM Dulbecco Telethon Institute International Mobility for Postdoctoral Research Training (DTI-IMPORT) and Spanish MINECO through Severo Ochoa Excellence Accreditation 2014-2018 (SEV-2013-0319) for the financial support. The research leading to these results has received funding also from "la Caixa" Foundation. We are also grateful to Dr. Fernando Bravo (ICIQ CSOL technology unit), Dr. Mihaela Delcea and Dr. Raghavendra Palankar (ZIK HIKE University of Greifswald) and Dr. Rossella Venditti (TIGEM). 


\section{References}

1. L. Hu, Z. W. Mao and C. Y. Gao. J. Mater. Chem, 2009, 19, 3108.

2. D. Peer, J. M. Karp, S. Hong, O. C. Farokhzal, R. Margalit and R. Langer. Nat. Nanotechnol. 2007, 2,751 .

3. H. Lord and S. O. Kelley. J. Mater. Chem, 2009, 19, 3127.

4. G. J. Nohyek, J. Lademann, C. Ribaud and M. S. Roberts. Crit. Rev. Toxicol, 2007, 37, 251.

5. S. K. Shukla. Adv. Mater. Rev, 2014, 1 (1), 2.

6. S. J. Soenen, B. B. Manshian, T. Aubert, U. Himmelreich, J. Demeester, S. C. De Smedt, Z. Hens and K. Braeckmans. Chem. Res. Toxicol., 2014, 27 (6), 1050.

7. P. G. Luo, S. Sahu, S. T. Yang, S. K. Sonkar, J. Wang, H. Wang, G. E. LeCroy, L. Cao and Y. P. Sun. J. Mater Chem B, 2013, 1, 2116.

8.Q. Liang, W. Ma, Y. Shi, Z. Li and X. Yang. Carbon, 2013, 60, 421.

9. M. Bacon, S. J. Bradley and T. Nann. Part. Part. Syst. Charact. 2014, 31, 415.

10. Q. Ma, I. Castello Serrano and E. Palomares. Chem. Comm., 2011, 47, 7071.

11. L. Li W. Gu, J. Liu, S. Yan and Z. P. Xu. Nano. Research, 2015, 8, 682.

12. K. Ladewig, Z. P. Xu and G. Q. Lu. Expert Opin Drug Deliv, 2009, 6 (9), 907.

13. K. Fent, C. J. Weisbrod, A. Wirth-Heller and U. Pieles. Aquatic toxicology, 2010, 100, 218.

14. Q. Mu, N. S. Hondow, L. Krzeminski, A. P. Brown, L. J. C. Jueken and M. N. Routledge. Particle and Fibre Toxicology, 2012, 9, 29.

15. I. L. Hsiao, A. M. Gramatke, R. Joksimovic, M. Sokolowski, M. Gradzielski and A. Haase. J. Nanomed. Nanotechnol, 2014, 5, 6.

16. Z. P. Xu, Q. H. Zeng, G. Q. Lu and A. B. Yu. Chem Eng Sci 2005, 61 (3) 1027.

17. J. H. Choy, S. Y. Hwak, Y. J. Jeong and J. S. Park. Angwe Chemie Int Ed, 2000, 39 (22), 4041.

18. J. M. Oh, S. J. Choi, G. E. Lee, J. E. Kim and J. H. Choy. Chem Asian J, 2009, 4, 67.

19. J. M. Oh, s. J. Choi, S. T. Kim and J. H. Choy. Bioconjugate Chem, 2006, 17, 1411.

20. G. Stoica, I. Castello Serrano, A. Figuerola, I. Ugarte, R. Pacios and E. Palomares. Nanoscale 2012, 4, 5409 .

21. B. Koean, Chem Rev, 2009m 109, 4283.

22. I. Castello Serrano, G. Stoica, A. Figuerola and E. Palomares. J Mater Chem B. 2013, 1, 793.

23. S. Cho, S. Jung, S. Jeong, J. Bang, J.; Park, Y. Park and S. Kim. Langmuir, 2013, 29 (1), 441.

24. M. P. Desai, V. Labhasetwar, E. Walter, R. J. Levy and G. L. Amidon. Pharma Res, 1997, 14, 1568 .

25. S. Prahba, W. Z. Zhou, J. Panyam and V. Labhasetwar, V. Int J Pharmacol, 2002, 244, 105.

26. C. Chen, P. Wang, T. T. Lim, L. Liu, S. Liu and R. Xu. J. Mater. Chem A, 2013, 1, 3877.

27. I. Castelló Serrano, Q. Ma and E. Palomares. J. Mater. Chem., 2011, 21, 17673.

28. N. Iyi, Y. Ebina and T. Sasaki, J. Mater. Chem., 2011, 21, 8085.

29. Y. Wang, Y. Zhou, T. Zhang, M. He and X. Bu, RSC Adv., 2014, 4, 29968.

30. F. Lu, S. H. Wu, Y. Hung and C. Y. Mou. Small 2009, 5 (12), 1408.

31. L. Treuel, X. Jiang and G. U. Nienhaus. J. Royal Society, 2013, 10 (82), 20120939. 
32. Z. P. Xu, Q. H. Zeng, G. Q. Lu and A. B. Yu. Chem Eng Sci 2005, 61 (3) 1027.

33. I. Passagne, M. Morille, M. Rousset, I. Pujalte and B. L'azou. Toxicol. 2012, 299, 112.

34. J. Blechinger, A. T. Bauer, A. A. Torrano, C. Gorzelanny, C. Bräuchle and S. W. Schneider. Small. 2013, 9 (23), 3970.

35. J. Patel and A. Patel. Toxicity of Nanomaterials on the Liver, Kidney, and Spleen. Biointeractions of Nanomaterials (Ed. B . Sutariya and Y. Pathak) CRC Press, 2014, p285-314. 